\title{
Revisão do gênero Corystes Mulsant (Coleoptera, Coccinellidae, Hyperaspinae, Hyperaspini) ${ }^{1}$
}

\author{
Julianne Milléo ${ }^{2}$ \\ Lúcia Massutti de Almeida ${ }^{2}$
}

\begin{abstract}
Review of the genus Corystes Mulsant (Coleoptera, Coccinellidae, Hyperaspinae, Hyperaspini). The genus Corystes Mulsant, 1850 is revised and illustrations of pertinent characters are provided. Lectotypes are designated for Diazonema fallax Weise, Callanga, Peru and Corystes cavifrons Weise, Turrialba, Costa Rica. Diazonema Weise, 1926 and Corystes (Diazonema) Duverger, 1989, are synonymyzed with Corystes Mulsant, and Diazonema fallax Weise, 1899 Corystes cavifrons Weise, 1926 and Corystes pubescens Weise, 1926 are placed in synonymy of Corystes hypocrita Mulsant, 1850.

KEY WORDS. Coccinellidae, Hyperaspinae, Hyperaspini, Corystes, taxonomy
\end{abstract}

Corystes Mulsant, 1850 foi descrito baseado em única espécie, Corystes hypocrita Mulsant, 1850, dentro do grupo "Hyperaspiens" sendo Corystes considerado próximo aos "Chilocoriens" por apresentar o clípeo expandido lateralmente, porém com uma saliência que o diferencia dos Chilocorini. Esse comentário de Mulsant confundiu os autores subseqüentes e por isso o gênero tem sido colocado em diferentes tribos. do Sul.

Este gênero têm distribuição restrita à América Central e norte da América

Para a revisão, o material-tipo foi estudado, com exceção de Corystes hypocrita, e os caracteres morfológicos, em especial a genitália masculina e feminina, foram reavaliados.

O material utilizado foi cedido para estudo pelas seguintes instituições: (BMNH) The Natural History Museum, Londres, Inglaterra; (NREA) Naturhistoriska Riksmuseet, Stockholm, Suécia; (UMZC) University Museum of Zoology, Cambridge, Inglaterra; (ZMHU) Zoologisches Museum der Humboldt Universitaet, Berlim, Alemanha.

A terminologia adotada foi a de GORDON (1985) e PLAZA INFANTE (19791980) para interpretação da genitália e MARINONI \& ALMEIDA (1983) para venação alar.

1) Contribuição número 1143 do Departamento de Zoologia, Universidade Federal do Paraná.

2) Departamento de Zoologia, Universidade Federal do Paraná. Caixa Postal 19020, 81531-990 Curitiba, Paraná, Brasil. Bolsistas do CNPq. 


\section{Corystes Mulsant, 1850}

Figs 1-20

Corystes Mulsant, 1850: 506-507 (descrição). - Crotch, 1874: 208 (sistemática). - Chapuis, 1876: 249-250 (sistemática). - Gorham, 1894: 182-183 (sistemática). - Weise, 1904: 358. - Weise, 1926: 12-13 (comentário). - Korschefsky, 1932: 267-268 (catálogo). -Blackwelder, 1945: 448 (check-list). - Chapin, 1965: 229, 269-271 (sistemática). - Chapin, 1966: 280 (chave, gênero). Gordon, 1987: 26 (catálogo). - Fürsch, 1989: 7, 11. - Fürsch, 1990: 4, 9 (catálogo). - Duverger, 1989: 146 (sistemática). - Almeida \& Milléo, no prelo (sistemática).

Diazonema Weise, 1926: 12-13. - Korschefsky, 1931: 202 (check-list). - Blackwelder, 1945: 448 (catálogo). - Fürsch, 1989: 6, 12. - Fürsch, 1990: 4, 10 (catálogo). Syn.n.

Corystes (Diazonema): Duverger, 1989: 146.

Espécie-tipo de Corystes: Corystes hypocrita Mulsant, 1850, por monotipia; de Diazonema: Diazonema cavifrons Weise, 1926.

\section{Histórico}

O gênero Corystes Mulsant, 1850, foi descrito dentro dos "Hyperaspiens" incluindo somente uma espécie, C. hypocrita.

CROTCH (1874), ao revisar os Coccinellidae, redescreveu brevemente o gênero Corystes e C. hypocrita, indicando-a como espécie-tipo.

CHAPUIS (1876) colocou Corystes em "Chilocorites", por possuir características deste grupo, mas enfatizou a semelhança com "Hyperaspites", representando uma forma de transição.

GORHAM (1894) fez breve redescrição do gênero e da única espécie e comentou que os caracteres evidenciavam que era próximo de Chilocorus Leach, 1815 , pela estrutura do clípeo que divide parcialmente os olhos.

WEISE (1899) considerou as interpretações de CHAPUIS (1876) incorretas e afirma que Corystes apresenta características típicas de Hyperaspini. WeISE (1903) descreveu C. cavifrons, de Turrialba, Costa Rica diferenciando-a pela projeção na cabeça, diferente daquela de C. hypocrita. Em 1904, comentou que GoRHAM (1894) também cometeu um equívoco ao incluir Corystes em Chilocorini reafirmando que indubitavelmente o gênero pertence à tribo Hyperaspini. Em 1926, ao examinar $C$. hypocrita, evidenciou que alguns dos espécimens recebidos com esse nome possuiam duas projeções semelhantes a dentes na região frontoclipeal, muito alargadas na direção anterior, que encobriam a base das antenas, e estabeleceu um novo gênero Diazonema, no qual incluiu C. cavifrons e descreveu Diazonema fallax. No mesmo trabalho descreveu Corystes pubescens, do Equador.

KORSCHEFSKY (1931-1932) colocou o gênero Corystes, com duas espécies, C. hypocrita e C. pubescens, na tribo Chilocorini e Diazonema com duas espécies, D. cavifrons e D. fallax em Hyperaspini. BLACKWELDER (1945) arrolou as mesmas espécies nos gêneros, mas incluiu ambos na tribo Hyperaspini.

CHAPIN (1965) na revisão dos gêneros de Chilocorini fez um histórico de Corystes e reafirmou que o mesmo pertence a Hyperaspini e em 1966 colocou Corystes na chave de identificação dos gêneros da tribo Hyperaspini.

GORDON (1987) citou que o material-tipo de Corystes hypocrita provavelmente se encontra no Muséum d'Histoire Naturelle, Lyon, França.

FÜRSCH (1989) relacionou o gênero Corystes na tribo Chilocorini e Diazonema em Hyperaspini; em 1990, colocou ambos em Hyperaspini. 
DUVERGER (1989) separou o gênero em Corystes (Corystes), com a espécietipo C. hypocrita Mulsant, 1850 e Corystes (Diazonema), espécie-tipo C. cavifrons Weise, 1903 e os incluiu em Hyperaspini, Hyperaspinae.

ALMEIDA \& MILLÉO (no prelo) compararam os caracteres de Corystes com dois outros gêneros de Hyperaspini, Hinda Mulsant, 1850 e Hyperaspis Redtenbacher, 1844.

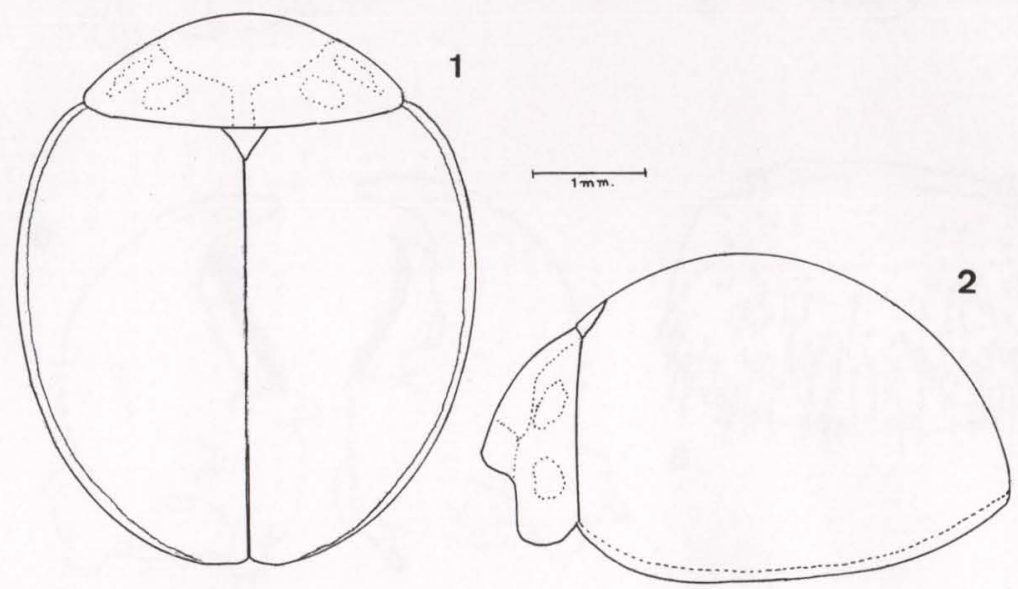

Figs 1-2. Corystes hypocrita. (1) Vista dorsal; (2) vista lateral.

\section{Redescrição}

Corpo em vista dorsal arredondado, robusto (Figs 1, 21) e fortemente convexo de perfil (Fig. 2).

Olhos finamente facetados e glabros, não emarginados pela fronte (Fig. 3). Inserção antenal oculta. Antenas curtas, com 9 artículos; escapo com grande lobo lateral, arredondado, com cerdas curtas e longas; pedicelo subquadrangular; os quatro artículos seguintes subquadrados, o antepenúltimo quatro vezes mais longo que largo, o penúltimo pequeno e quadrangular, e o último muito pequeno e triangular (Fig. 4). Fronte com região central profunda, muito côncava e prolongada desde o centro, onde se estreita até a região anterior dos olhos; bordas laterais expandidas, formando uma elevação em aba (Fig. 22). Clípeo com margem apical em arco côncavo e bordas laterais pouco expandidas por baixo da elevação da fronte. Labro parcialmente oculto, subquadrangular (Fig. 5). Mandíbulas grandes e robustas, fortemente esclerotinizadas; com dois dentes incisivos, apical e subapical e dente mediano molar direito terminando em ponta e esquerdo sub-quadrado; prosteca grande com cerdas largas (Fig. 6). Maxilas completas e esclerotinizadas; quarto artículo do palpo maxilar fortemente securiforme de lados divergentes (Fig. 7). Lábio com margem anterior da lígula, densamente coberta com pequenos espinhos e na porção ventral mediana com algumas cerdas longas; cada palpo labial com três artículos, o basal mais curto e o terceiro afilado na porção distal; mento com projeções apicais longas, com separação relativamente larga e cerdas longas nas bordas (Fig. 8). 

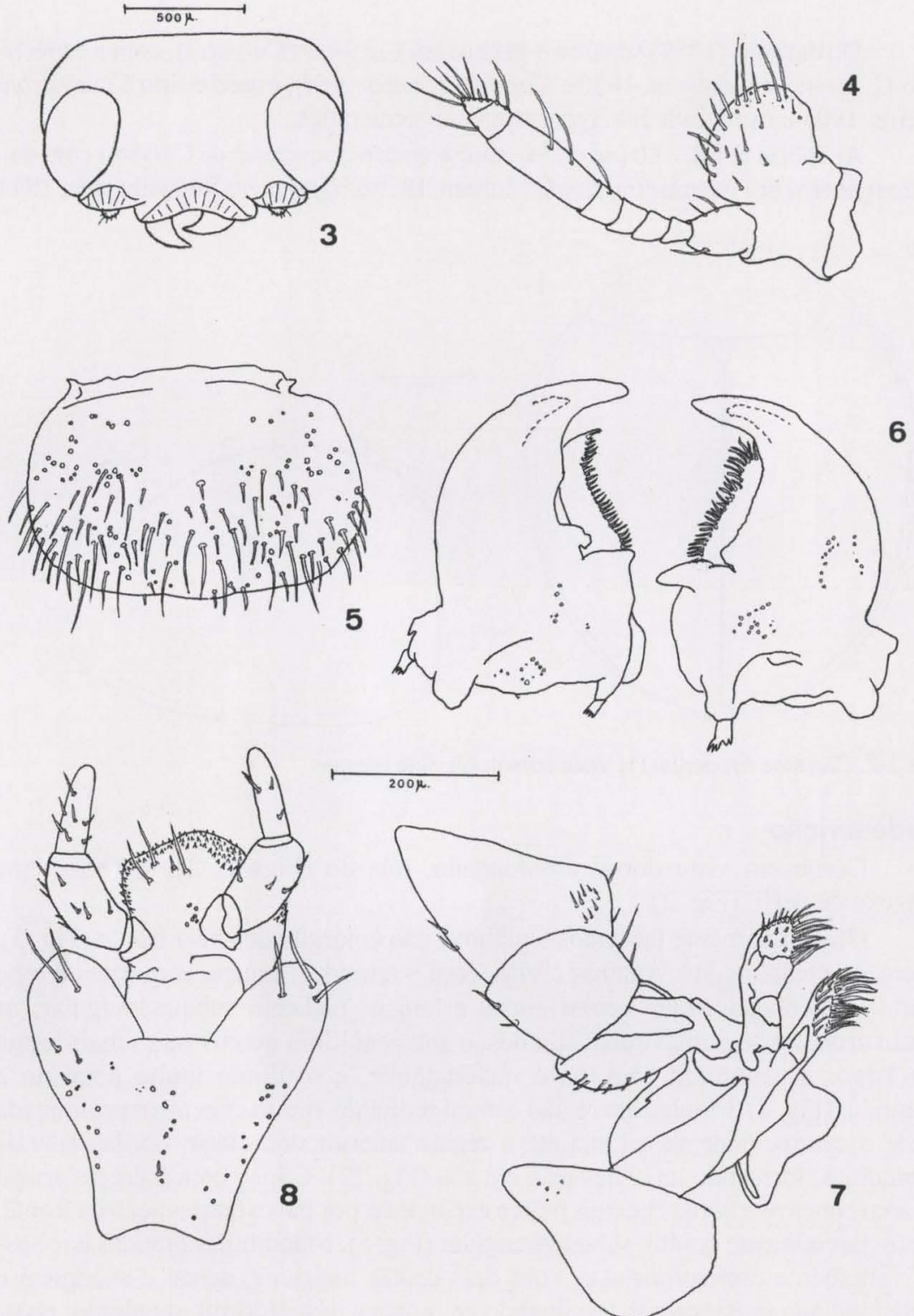

Figs 3-8. Corystes hypocritant. (3) Cabeça vista frontal; (4) antena; (5) labro; (6) mandíbulas vista dorsal; (7) maxila; (8) lábio.

Pronoto com máculas distintas. Carenas do prosterno convergentes, mais curtas que o processo prosternal; este com lados projetados e com uma constricção para em seguida se alargar terminando em forma de seta de bordos arredondados (Fig. 9). 
Metendosternito trapezoidal, com bordos laterais retos e bordo anterior em "V", deixando os tendões mais afastados; braços laterais com ápice curvado para fora (Fig. 10).

Venação alar conforme o padrão de Coccinellidae, com pequena variação da 1A, pouco definida na sua porção basal (Fig. 11).

Élitros arredondados com calo umeral pouco saliente e margem lateral fortemente esplanada (Fig. 1). Epipleura elitral larga, com forte escavação para receber o ápice dos fêmures médio e posterior (Fig. 12).

Fêmur robusto, profundamente escavado para recepção da tíbia. Todas as tíbias dilatadas, com forte ângulo externo, escavação muito profunda para recepção do tarso até o ângulo externo (Fig. 13). Segundo tarsômero em vista ventral, subtriangular (Fig. 15); garras tarsais com dente basal subquadrado (Fig. 14).

Linha pós-coxal incompleta sobre o primeiro esterno abdominal. Abdome do macho (Fig. 16) e da fềmea sem modificações, ambos com apenas seis segmentos visíveis.

Genitália do macho: Lobo médio assimétrico, alargado na base com cerdas na região mediana e ápice estreito e arredondado. Parâmeros mais longos que o lobo médio; com densa fileira de cerdas longas marginais e cerdas mais curtas internas (Fig. 17). Sifão fortemente esclerotinizado e curvado (Figs 18, 19).

Genitália da fềmea. Espermateca globular, com placas coxais transversas, coxitos largos e subquadrados com longas cerdas e sem estilos (Fig. 20).

\section{Discussão taxonômica}

Este gênero tem sido motivo de discussão desde que CHAPUIS (1876) o incluiu em "Chilocorites" e GoRHAM (1874) evidenciou os caracteres semelhantes a Chilocorus Leach, 1815. Entretanto, Corystes apresenta caracteres marcantes que o inclui em Hyperaspini, tais como: processo prosternal com carenas, epipleura do élitro escavada para recepção do ápice do fêmur médio, linha pós-coxal incompleta sobre o primeiro esterno abdominal, genitália do macho com os parâmeros ligados diretamente ao lobo médio, genitália da fêmea com placas coxais transversas e espermateca globular com corno separado do nódulo por um longo tubo e bolsa copulatória sem infundíbulo. Além disso, os Chilocorini possuem o clípeo projetado para os lados dividindo completamente os olhos, enquanto em Corystes, o clípeo tem forma de aba dividida, de maneira que uma parte se sobrepõem ao labro e as duas laterais se projetam sobre a inserção antenal (Fig. 3).

Corystes foi incluido na tribo Hyperaspini por DUVERGER (1989), em função da espermateca globular e sem infundíbulo, junto aos gêneros Hyperaspis e Thalassa Mulsant, 1850. Em Corystes o corpo é muito convexo e arredondado, epipleura esplanada lateralmente; inserção antenal oculta; clípeo com bordas laterais projetadas; antena com 9 artículos; escapo com projeção lateral grande e pronunciado; processo prosternal com constrição basal; tíbia anterior com o bordo maior que $90^{\circ}$ e com projeção foliácea; seis segmentos abdominais visíveis no macho e na fêmea. Corystes apresenta semelhanças com outros gêneros de Brachiacanthadini que também pertence a subfamilia Hyperaspinae. Pode ser comparado com Brachiantha Chevrolat, 1849, que apresenta tíbia anterior com espinho muito afilado e ainda com Hinda e Cyra Mulsant, 1850 (MiLLÉo et al. 1997) por possuirem antena com 11 artículos, sem lobo lateral no escapo e genitália da fêmea com espermateca tubular, com infundíbulo. 
Tendo em vista que a genitália masculina e feminina das quatro espécies incluídas em Corystes e em Diazonema apresentam-se morfologicamente idênticas, D. cavifrons, $D$. fallax e C. pubescens foram sinonimizadas com Corystes hypocrita Mulsant, 1850.

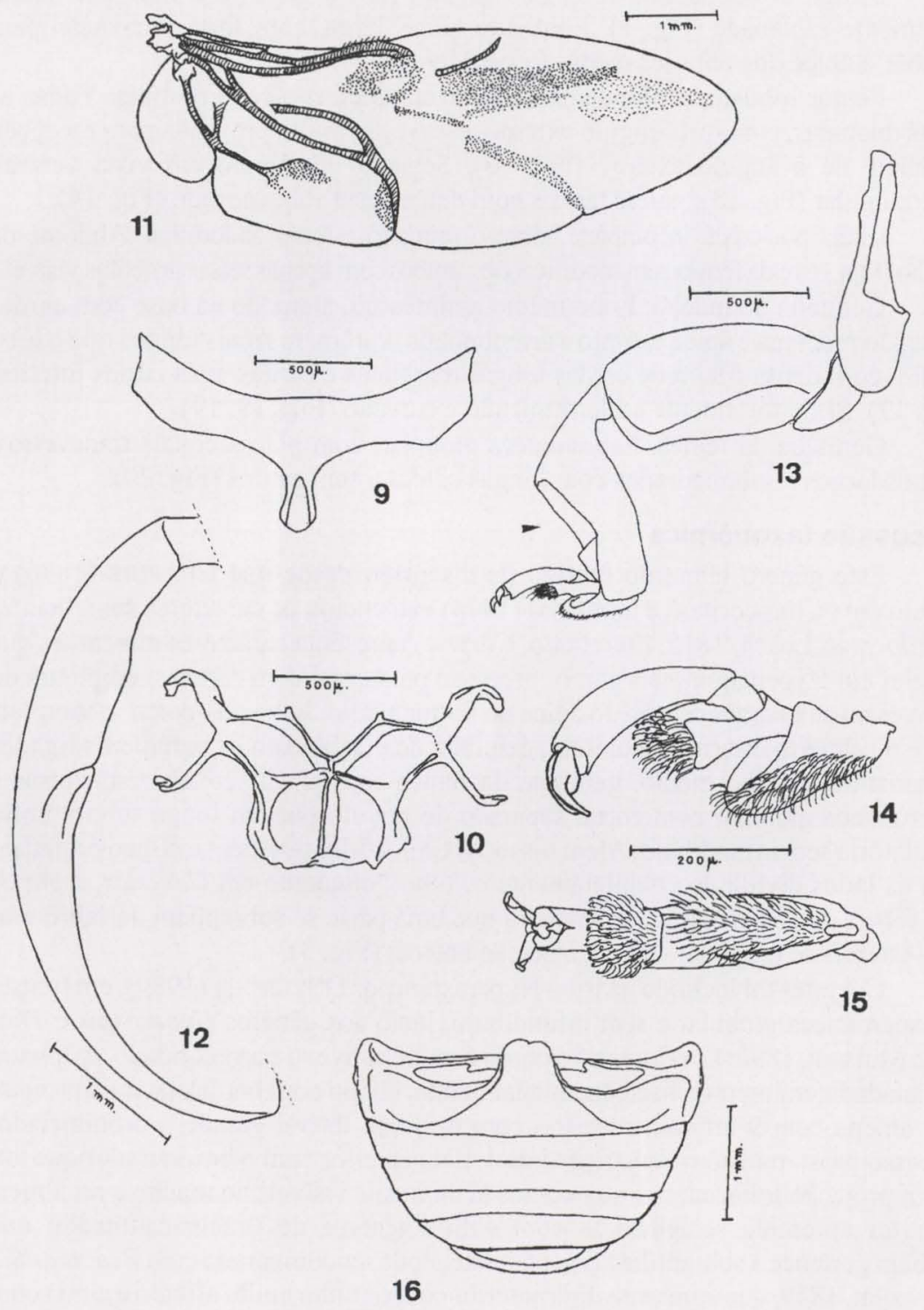

Figs 9-16. Corystes hypocrita. (9) Prosterno; (10) metendosternito; (11) asa posterior; (12) epipleura vista ventral; (13) perna anterior; (14) tarso anterior vista lateral; (15) tarso anterior vista ventral; (16) abdome do macho vista ventral. 


\section{Corystes hypocrita Mulsant, 1850}

Figs 21-23

Corystes hypocrita Mulsant, 1850: 507 (descrição). - 1853: 197-198 (redescrição). - Crotch, 1874: 208 (sistemática). - Weise, 1899: 269. - 1903: 269 (sistemática). - 1926: 12-13. - Korschefsky, 1932: 267-268 (catálogo). - Blackwelder, 1945: 448 (check-list). - Chapin, 1965: 270 (sistemática). Gordon, 1987: 26 (catálogo). - Fürsch, 1989: 11. - Fürsch, 1990: 9 (catálogo).

Corystes cavifrons Weise, 1903: 208 (descrição). Syn.n.

Diazonema cavifrons: Weise, 1926: 12-13 (sistemática). -Korschefsky, 1931: 202 (catálogo). Blackwelder, 1945: 448 (check-list), - Fürsch, 1989: 12, - Fürsch, 1990: 10 (catálogo).

Diazonema fallax Weise, 1926: 13 (descrição). - Korschefsky, 1931: 202 (catálogo). - Blackwelder, 1945: 448 (check-list). - Chapin, 1965: 270 (sistemática). Syn.n.

Corystes pubescens Weise, 1926: 34 (descrição). - Korschefsky, 1932: 268 (catálogo). - Blackwelder, 1945: 448 (check-list). Syn.n.

Corystes (Corystes) hypocrita: Duverger, 1989: 146 (sistemática).

Corystes (Diazonema) cavifrons: Duverger, 1989: 146. (sistemática).

Diagnose. Corpo com formato arredondado e fortemente convexo. Comprimento: 4,20 a 4,68 $\mathrm{mm}$. Largura: 3,84 a 4,20 $\mathrm{mm}$. Pronoto e élitros vermelho-alaranjados, contornados por uma estreita faixa marrom. Pronoto com quatro máculas variando de marrom-claro a amarelo: duas maiores circulares laterais e duas ovalares estreitas oblíquas separadas na base por aproximadamente a largura de cada uma delas e anteriormente divergentes (Fig. 24). Superfície ventral castanha.

Inserção antenal oculta. Clípeo com margem apical côncava e bordas laterais elevadas e pronunciadas, transparentes, dando aspecto de aba (Fig. 22). Olhos finamente facetados e glabros, largamente emarginados pela fronte (Fig. 3). Antenas curtas, com nove artículos; escapo arredondado com grande lobo lateral. Processo prosternal em forma de seta com borda arredondada. Todas as tíbias dilatadas, com forte ângulo externo, escavação muito profunda para recepção do tarso até o ângulo externo. Linha pós-coxal incompleta. Abdome do macho e da fêmea com seis segmentos visíveis.

Variações intraespecíficas. As máculas do pronoto podem estar todas mais ou menos fundidas (Fig. 21). Os espécimens provenientes da Costa Rica (descritos por Weise como $D$. cavifrons) apresentam a fronte muito mais escavada, côncava e as bordas laterais muito elevadas em projeções semelhantes a dentes, alargadas na base, encobrindo a inserção das antenas (Figs 23-24). O espécimen do Equador descrita por Weise como C. pubescens, apresenta no pronoto e élitros densas cerdas curtas amarelo-douradas (Fig. 25).

Distribuição. Nicarágua, Costa Rica, Guiana Francesa, Equador, Peru e Brasil.

Material-tipo. Holótipo de Corystes hypocrita, procedente de Caiena, Guiana Francesa (não examinado). GoRDON (1987) indicou que, provavelmente estaria no Muséum d'Histoire Naturelle, Lyon, França. O material não foi localizado no referido museu.

Holótipo de Corystes pubescens, procedente do Equador, foi examinado e encontra-se depositado no NREA.

Diazonema fallax procedente de Callanga, Peru, está depositado no ZMHU. Os dados de seis exemplares recebidos conferem com os indicados por WEISE (1926). Um dos exemplares possui ainda uma etiqueta manuscrita indicando que o mesmo é o exemplar-tipo. Portanto designa-se esse exemplar como lectótipo. 


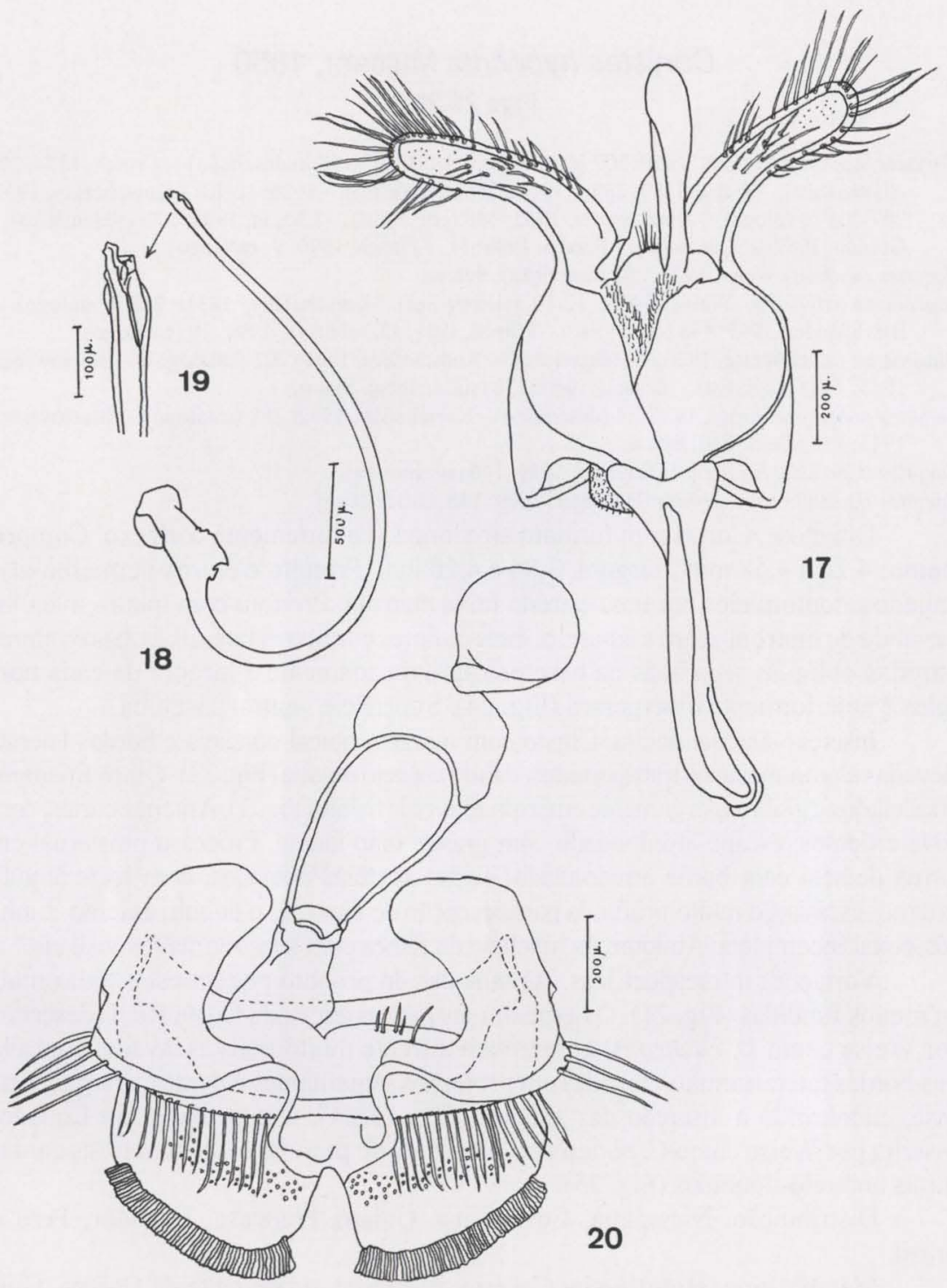

Figs 17-20. Corystes hypocrita, genitália do macho. (17). Tégmen; (18) sifão; (19) ápice do sifäo; (20) genitália da fêmea.

Corystes cavifrons, procedente de Turrialba, Costa Rica, está depositado no ZMHU. Foram examinados dois exemplares com dados de procedência que conferem com os apresentados por WEISE (1903). Ambos possuem as seguintes etiquetas: "Costa Rica"; "Collection Schild-Burgdorf, Costa Rica, Turrialba"; "Zool. Mus. Berlim". Portanto designa-se o exemplar com etiqueta manuscrita "Corystes cavifrons $\mathrm{m} . "$ como lectótipo. 

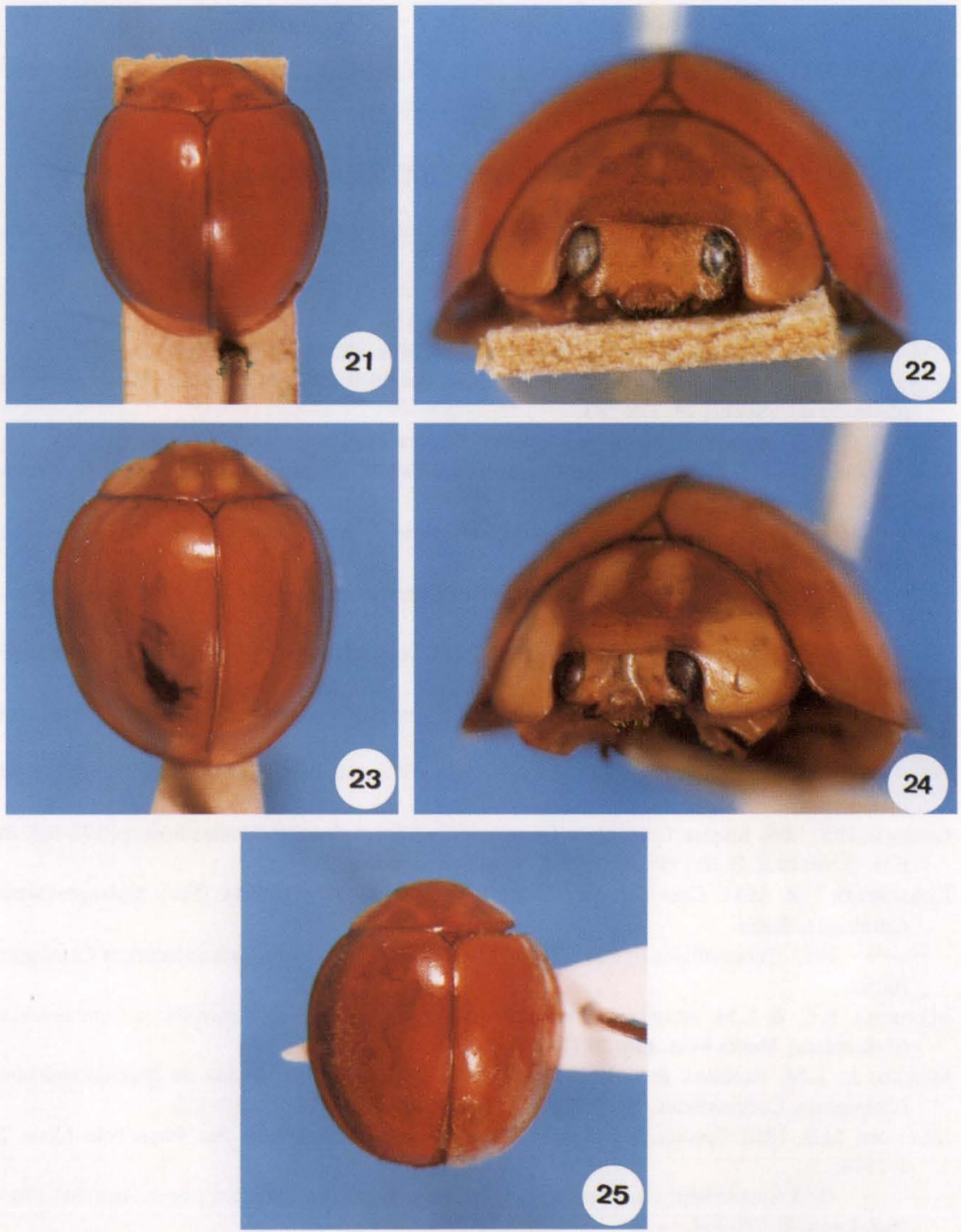

Figs 21-25. Corystes hypocrita. (21) Exemplar do Peru, vista dorsal, (22) vista frontal; (23) exemplar da Costa Rica vista dorsal, (24) vista frontal; (25) exemplar do Equador, vista dorsal.

Material-examinado. Costa RicA. Cartago: Turrialba, 2 exs., col. SchildBurgdorf (ZMHU); Tucurrique, 1 ex., col. Schild-Burgdorf (ZMHU), Alajuela, San Carlos, 1 ex., col. Schild-Burgdorf(ZMHU). EQUADOR. Dr. Ohaus leg., 1 ex. (NREA). PERU. 1 ex. (ZMHU); Cuzco: Callanga, 6 exs. (ZMHU); Junín: Rio Oxabamba, 1 ex. (ZMHU). Brasil. Amazonas: Tefé (Ega), 1 ex. (UMZC); 1 ex., col. Fry (BMNH); Pará: Santarém, 1 ex. (UMZC). Sem procedência, 1 ex., col. Deyrolle (UMZC); 1 ex., Blackwelder leg. (UMZC). 
AGRADECIMENTOS. À Dra. Danúncia Urban pela revisão crítica do texto e ao Dr. Albino Morimasa Sakakibara pelas fotografias dos exemplares.

\section{REFERÊNCIAS BIBLIOGRÁFICAS}

Almeida, L.M. \& J. Milléo. no prelo. A Review of the Genus Hinda Mulsant (Coleoptera, Coccinellidae). Coleopterists Bull. .

BLACKWELDER, R.E. 1945. Checklist of the coleopterous insects of Mexico, Central America, the West Indies, and South America. Bull. U.S. Nat. Mus. 185 (3): 343-550.

ChapIN, E.A. 1965. The genera of the Chilocorini. Bull. Mus. Comp. Zool. 133 (4): 227-271.

. 1966. A new species of myrmecophilous Coccinellidae with notes on other Hyperaspini (Coleoptera). Psyche. 73: 278-283.

ChApUIS, F. 1876. Histoire naturelle des insectes. Genera des Coléoptères, Paris, 12, p. 1-424.

СRотсH, G.R. 1874. A Revision of the Coleopterous Family Coccinellidae. London, E.W. Janson, $311 \mathrm{p}$.

Duverger, C. 1989. Contribution à l'etude de Hyperaspinae $1^{\text {ère }}$ note (Coleoptera, Coccinellidae). Bull. Soc. Linn. Bordeaux 17 (3): 143-157.

FürsCH, H. 1989. Coccinella. Newsletter for Systematic research in Coccinellids. Universitàt Passau $1(1): 1-42$.

. 1990. Coccinella. Newsletter for Systematic research in Coccinellids. Universitàt Passau 2 (1): $1-40$.

Gordon, R.D. 1985. The Coccinellidae (Coleoptera) of America North of Mexico. Jour. N.Y. Entomol. Soc. 93 (1): 352-599.

Ent. 3: 1-46.

Gorham, H.S. 1894. Insecta. Coleoptera. Erotylidae, Endomychidae and Coccinellidae, p. 177-208. In:

F.D. Godman \& O. Salvin. Biologia Centrali-Americana 7.

Korschefsky, R. 1931. Coccinellidae I. Part 118, p. 1-224. In: W. JunK (Ed.). Coleopterorum Catalogus. Berlin.

- 1932. Coccinellidae II. Part 120, p. 225-659. In: W. JUNK. (Ed.) Coleopterorum Catalogus. Berlin.

MARINoni, R.C. \& L.M. AlmeidA. 1983. Sobre a venação alar em Coccinellidae e Cerambycidae (Coleoptera). Revta bras. Ent. 27 (3/4): 267-275.

Milléo, J.; L.M. AlmeidA \& I.M.M. LiMA. 1997. Contribuição do estudo de Brachiacanthadini (Coleoptera, Coccinellidae, Hyperaspinae). Revta bras. Zool. 14 (2): 391-405.

Mulsant, M.E. 1850. Species de Coléoptères trimères sécuripalpes. Ann. Sci. Phys. Nat. Lyon. 2: $1-1104$.

.1853. Supplément a la Monographie des Coléoptéres Triméres Sécuripalpes. Ann. Sci. Phys. Nat. Lyon. 2: 129-334.

PLAZA INFANTE, E. 1979-1980. Morfología y nomenclatura de la genitalia de los Coccinellidae. Graellsia 35-36: 85-101.

WEISE, J. 1899. Coccinelliden aus Süd-Amerika. Dtsch. ent. Zeitschr. 2: 257-272

- 1903. Drei neue Coccinellen. Dtsch. ent. Zeitschr.: 207-208.

1904. Synonymische Bemerkungen zu Gorham, Biologia Centrali-Americana, Coccinellidae. Dtsch. ent. Zeitschr. 3: 357-364.

1926. Uber bekannte und new Chrysomeliden und Coccinelliden aus dem Reich museum zu Stockholm. Ark. Zool. 18 (34): 1-34.

Recebido em 15.IV.1999; aceito em 01.II.2000. 